\title{
CMB constraints on Dark Matter models with large annihilation cross-section
}

\author{
Silvia Galli ${ }^{a, b}$, Fabio Iocco $^{c, d}$, Gianfranco Bertone $^{d}$, Alessandro Melchiorri ${ }^{b}$ \\ ${ }^{a}$ Laboratoire Univers et Théorie (LUTH), Observatoire de Paris, \\ Meudon, Université Paris Diderot - 75205 PARIS \\ ${ }^{b}$ Physics Department and INFN, Universita' di Roma "La Sapienza", Ple Aldo Moro 2, 00185, Rome, Italy \\ ${ }^{c}$ Institut de Physique Théorique, CNRS, URA 2306 \& CEA/Saclay, F-91191 Gif-sur-Yvette and \\ ${ }^{d}$ Institut d'Astrophysique de Paris, UMR 7095-CNRS Paris, \\ Université Pierre et Marie Curie, boulevard Arago 98bis, 75014, Paris, France.
}

(Dated: April 30, 2009)

\begin{abstract}
The injection of secondary particles produced by Dark Matter (DM) annihilation around redshift $\sim 1000$ would inevitably affect the process of recombination, leaving an imprint on Cosmic Microwave Background (CMB) anisotropies and polarization. We show that the most recent CMB measurements provided by the WMAP satellite mission place interesting constraints on DM selfannihilation rates, especially for models that exhibit a large Sommerfeld enhancement of the annihilation cross-section, as recently proposed to fit the PAMELA and ATIC results. Furthermore, we argue that upcoming CMB experiments such as Planck, will improve the constraints by at least one order of magnitude, thus providing a sensitive probe of the properties of DM particles.
\end{abstract}

PACS numbers: 26.35.+c, 98.80.Cq, 98.80.Ft

SACLAY-T09/046

\section{INTRODUCTION}

The recent measurements of the Cosmic Microwave Background (CMB) anisotropy and polarization from experiments as WMAP [1], ACBAR [2] and BOOMERANG [3] have confirmed the theoretical predictions of the standard cosmological model based on inflation, dark matter and a cosmological constant. This not only permits to place stringent constraints on several parameters of the model but also to use it as new laboratory where to test physical processes in a environment not achievable otherwise.

In particular, there is a remarkable agreement between the theoretical description of the recombination process, occurring at $z_{r} \sim 1000$, and CMB data, a circumstance that severely constrains new sources of ionizing photons, and more in general any deviation from standard recombination [4], as recently shown by several groups of authors (see e.g. [5], 6], 7]). Most of the recent literature has analyzed the modified recombination by means of a phenomenological approach, parameterizing in a model independent way the modifications to the recombination process. Here, we focus instead on the constraints that can be placed on the properties of DM particles, under the assumption that recombination is modified only by dark matter annihilation. With respect previous studies [8, 9], our analysis includes more recent data (WMAP 5 -year data), and it concentrates on a new class of DM models that have been recently proposed to explain the observed anomalies in cosmic ray data.

In fact, the attempt to explain the high energy positron and electron rise seen by PAMELA [10] and ATIC [11] in terms of Dark Matter (DM) annihilation has prompted the proliferation of new DM candidates with very large annihilation cross-section. In particular, in models with a "Sommerfeld" enhancement of the annihilation crosssection $(\sigma v)$, the efficent exchange of force carriers at low relative particle velocities leads to a velocity-dependent $(\sigma v)$, which behaves roughly as $\propto 1 / v$ for high $v$, and saturates below a critical $v_{s}$ (typically smaller than the local velocity dispersion, $v_{\odot}$, see below), that depends on the ratio between the masses of the force carrier and the DM particle. A nice feature of these models is that they can be made naturally consistent with standard thermal freeze-out. In fact, DM freezes out typically with $\beta \equiv v / c=\mathcal{O}(1)$, and $(\sigma v)$ will grow from this minimum value as the universe cools and expand. Then, when the first gravitationally bound structures form, DM virializes within the gravitational potential of the host halo, thus leading for Milky Way (MW)-like galaxies at $\mathrm{z}=0$ to virialized velocities of order $\beta \sim 10^{-3}$. Smaller velocities, thus a larger $(\sigma v)$ can be achieved in DM haloes with low velocities such as MW subhaloes 12 or small haloes at high redshift [13]. although it is unclear whether the annihilation flux can be boosted enough to explain the PAMELA and ATIC data without being in conflict with other measurements, such as the anti-proton or gammaray fluxes towards the Galactic center (see e.g. 14] and references therein).

When recombination occurs, around $z_{r} \sim 1000$, the relic WIMPs have not yet formed sizable gravitationally bound structures and are cold enough for the Sommerfeld mechanism to produce substantial enhancement of the annihilation cross-section with respect to the thermal value (after kinetic decoupling DM particle temperature evolves adiabatically as $\mathrm{T} \propto z^{2}$, so $\beta\left(z_{r}\right) \sim 10^{-8}$, for a $\mathcal{O}\left(100 \mathrm{GeV} / \mathrm{c}^{2}\right)$ mass WIMP $)$. As we will see below, the actual enhancement is model-dependent, because different DM models lead to a different behaviour of $(\sigma v)_{z}$, but in general we expect that for large enough cross-sections, DM annihilation will significantly modify the recombination history, thus leaving a clear imprint on the angular power spectra of CMB anisotropy and polarization.

Our paper is organized as follows: in the next section 
we describe the effects of annihilating dark matter on primordial recombination and the characteristic imprints on the CMB angular spectra. In section III we describe our analysis method. In section IV we present the results of our analysis. Finally, in Section V, we discuss our conclusions.

\section{ANNIHILATING DARK MATTER AND THERMAL HISTORY OF THE UNIVERSE}

Annihilating particles affect the ionization hystory of the Universe in three main different ways. The interaction of the shower produced by the annihilation with the thermal gas can $i$ : ionize it, $i i$ : induce $\mathrm{Ly}-\alpha$ excitation of the hydrogen and $i i i$ : heat the plasma; the first two modify the evolution of the free electron fraction $x_{e}$, the third affects the temperature of baryons. In the presence of annihilating particles, the evolution of the ionization fraction $x_{e}$ satisfies:

$$
\frac{d x_{e}}{d z}=\frac{1}{(1+z) H(z)}\left[R_{s}(z)-I_{s}(z)-I_{X}(z)\right],
$$

where $R_{s}$ is the standard recombination rate, $I_{s}$ the ionization rate by standard sources, and $I_{X}$ the ionization rate due to particle annihilation.

Following the seminal papers [15], standard recombination is described by:

$$
\left[R_{s}(z)-I_{s}(z)\right]=C \times\left[x_{\mathrm{e}}^{2} n_{\mathrm{H}} \alpha_{\mathrm{B}}-\beta_{\mathrm{B}}\left(1-x_{\mathrm{e}}\right) \mathrm{e}^{-h_{\mathrm{P}} \nu_{2 s} / k_{\mathrm{B}} T_{\mathrm{b}}}\right]
$$

where $n_{H}$ is the number density of hydrogen nuclei, $\alpha_{\mathrm{B}}$ and $\beta_{\mathrm{B}}$ are the effective recombination and photoionization rates for principle quantum numbers $\geq 2$ in Case B recombination, $\nu_{2 s}$ is the frequency of the $2 s$ level from the ground state and $T_{b}$ is the temperature of the baryonic gas and the factor $C$ is given by:

$$
C=\frac{\left[1+K \Lambda_{2 s 1 s} n_{\mathrm{H}}\left(1-x_{\mathrm{e}}\right)\right]}{\left[1+K \Lambda_{2 s 1 s} n_{\mathrm{H}}\left(1-x_{\mathrm{e}}\right)+K \beta_{\mathrm{B}} n_{\mathrm{H}}\left(1-x_{\mathrm{e}}\right)\right]}
$$

where $\Lambda_{1 s 2 s}$ is the decay rate of the metastable $2 s$ level, $n_{\mathrm{H}}\left(1-x_{e}\right)$ is the number of neutral ground state $H$ atoms, and $K=\lambda_{\alpha}^{3} /(8 \pi H(z))$ with $H(z)$ the Hubble expansion factor at redshift $z$ and $\lambda_{\alpha}$ is the wavelength of the Ly- $\alpha$ transition from the $2 p$ level to the $1 s$ level.

The $I_{X}$ term of equation 1 represents the contribute to the electron fraction evolution by a "non-standard" source; in our case it takes into account that during recombination particle annihilation increases the ionization rate both by direct ionization from the ground state, and by contributing additional Lyman- $\alpha$ photons. The latter boosts the population at $n=2$, increasing the rate of photoionization by the CMB from these excited states. Therefore, the ionization rate due to particle annihilation is:

$$
I_{X}(z)=I_{X i}(z)+I_{X \alpha}(z)
$$

where $I_{X i}$ is the ionization rate due to ionizing photons, and $I_{X \alpha}$ the ionization rate due to additional Lyman alpha photons.

The rate of energy release $\frac{d E}{d t}$ per unit volume by a relic self-annihilating dark matter particle is given by

$$
\frac{d E}{d t}(z)=\rho_{c}^{2} c^{2} \Omega_{D M}^{2}(1+z)^{6} f \frac{\langle\sigma v\rangle}{m_{\chi}}
$$

with $n_{D M}(z)$ being the relic DM abundance at a given redshift $z,\langle\sigma v\rangle$ is the effective self-annihilation rate and $m_{\chi}$ the mass of our dark matter particle, $\Omega_{D M}$ is the dark matter density parameter and $\rho_{c}$ the critical density of the Universe today; the parameter $f$ indicates the fraction of energy which is absorbed overall by the gas, under the approximation the energy absorption takes place locally. This on-the-spot approximation has been adopted by previous analysis ([9])

Each of the terms in Eq. 4 is related to the rate of energy release as:

$$
\begin{aligned}
I_{X i} & =C \chi_{i} \frac{[d E / d t]}{n_{H}(z) E_{i}} \\
I_{X \alpha} & =(1-C) \chi_{\alpha} \frac{[d E / d t]}{n_{H}(z) E_{\alpha}}
\end{aligned}
$$

where $E_{i}$ is the average ionization energy per baryon, $E_{\alpha}$ is the difference in binding energy between the $1 s$ and $2 p$ energy levels of a hydrogen atom, $n_{H}$ is the number density of Hydrogen Nuclei and $\chi_{i}=\chi_{\alpha}=\left(1-x_{e}\right) / 3$ are the fractions of energy going to ionization and to Lymanalpha photons respectively, given by [16] following the approach of Shull and Van Steenberg [17].

Finally, a fraction of the energy released by annihilating particles goes into heating of baryonic gas, adding an extra $K_{h}$ term in the standard evolution equation for the matter temperature $T_{b}$ :

$$
\begin{aligned}
(1+z) \frac{d T_{b}}{d z}= & \frac{8 \sigma_{T} a_{R} T_{C M B}^{4}}{3 m_{e} c H(z)} \frac{x_{e}}{1+f_{\mathrm{He}}+x_{e}}\left(T_{b}-T_{C M B}\right) \\
& -\frac{2}{3 k_{B} H(z)} \frac{K_{h}}{1+f_{\mathrm{He}}+x_{e}}+2 T_{b}
\end{aligned}
$$

where the non standard term is given by:

$$
K_{h}=\chi_{h} \frac{(d E / d t)}{n_{H}(z)}
$$

and $\chi_{h}=\left(1+2 x_{e}\right) / 3$ is the fraction of energy going into heat given by [16].

\section{ANNIHILATING DARK MATTER AND THE CMB}

We can now compute the theoretical angular power in presence of DM annihilations, by modifying the RECFAST routine ([18]) in the CAMB code (19]), following 


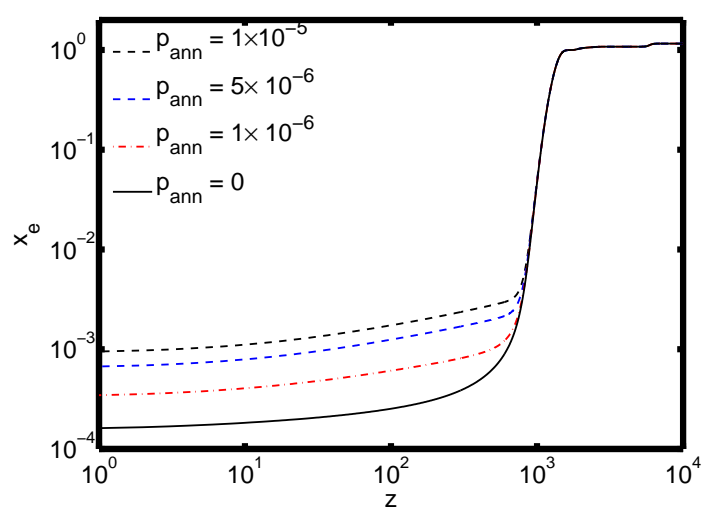

FIG. 1: Evolution of the free electron fraction as function of redshift for different values of $p_{a n n}=\left[0,10^{-6}, 5 \times 10^{-6}, 10^{-5}\right]$ $m^{3} / s / K g$.

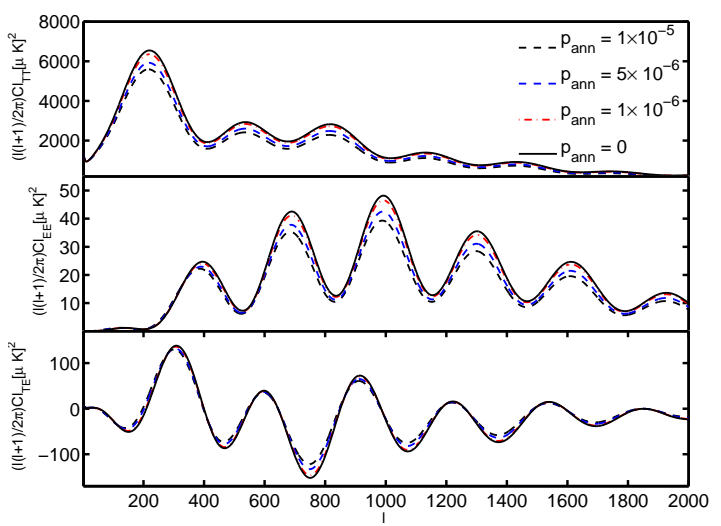

FIG. 2: TT, TE, EE angular power spectra (from Top to Bottom) for different values of $p_{a n n}=\left[0,10^{-6}, 5 \times 10^{-6}, 10^{-5}\right]$ $\mathrm{m}^{3} / \mathrm{s} / \mathrm{Kg}$.

the prescription described in the previous section. The dependence on the properties of the DM particles is encoded in the quantity

$$
f \frac{<\sigma v>}{m_{\chi}} \equiv p_{a n n}
$$

appearing in eq. 5. that we use as a parameter in the code.

In Fig 1 we show the evolution of the free electron fraction for different values of $p_{a n n}$. As we can see, the DM annihilation model we consider can increase the free electron fraction after $z \sim 1000$ by one order of magnitude, increasing the optical depth to last scattering surface and smearing the visibility function. The consequences of such annihilation can be seen in Fig, 2 where we show the CMB anisotropy, cross-polarization and polarization angular power spectra for different values of $p_{a n n}$. DM annihilation damps the acoustic oscillations in the angular power spectra as in the case of an instantaneous reionization. However, large scale polarization is left unchanged by dark matter annihilation and a degeneracy between these two effects can indeed be broken. Although DM
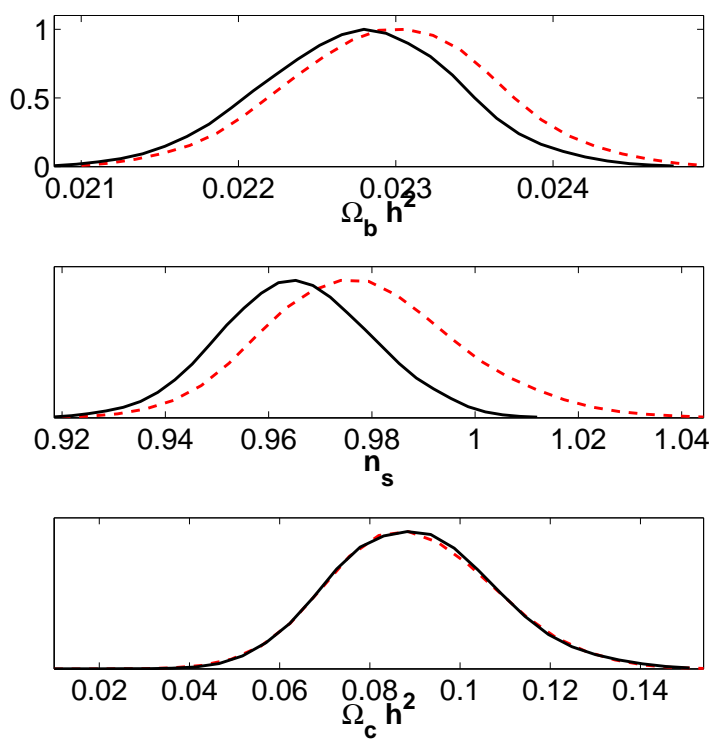

FIG. 3: Constraints on the $\omega_{b}, n_{s}$ and $\omega_{c}$ parameters in the case of standard recombination (solid line), or including dark matter annihilation (dashed line).

annihilation could play a role in the subsequent reionization of the Universe, the effect is likely to be small [20], unless one invokes very high anihilation cross sections [21]. Here, we don't consider a particular model for reionization, and simply adopt the parametrization of a full and instantaneous reionization at redshift $z_{r}<30$.

We search for an imprint of self-annihilating dark matter in current CMB angular spectra by making use of the publicly available Markov Chain Monte Carlo package cosmomc [22]. Other than $p_{a n n}$ we sample the following six-dimensional set of cosmological parameters, adopting flat priors on them: the physical baryon and CDM densities, $\omega_{b}=\Omega_{b} h^{2}$ and $\omega_{c}=\Omega_{c} h^{2}$, the scalar spectral index, $n_{s}$, the normalization, $\ln 10^{10} A_{s}(k=0.05 / M p c)$, the optical depth to reionization, $\tau$, and the ratio of the sound horizon to the angular diameter distance, $\theta$.

We consider purely adiabatic initial conditions. The MCMC convergence diagnostic tests are performed on 4 chains using the Gelman and Rubin "variance of chain mean" / "mean of chain variances" $R-1$ statistic for each parameter. Our $1-D$ and $2-D$ constraints are obtained after marginalization over the remaining "nuisance" parameters, again using the programs included

\begin{tabular}{rc} 
Experiment & $p_{\text {ann }} 95 \%$ c.l. \\
\hline WMAP $<2.0 \times 10^{-6} \mathrm{~m}^{3} / \mathrm{s} / \mathrm{kg}$ \\
\hline Planck $<1.5 \times 10^{-7} \mathrm{~m}^{3} / \mathrm{s} / \mathrm{kg}$ \\
\hline $\mathrm{CVl}<5.0 \times 10^{-8} \mathrm{~m}^{3} / \mathrm{s} / \mathrm{kg}$ \\
\hline
\end{tabular}

TABLE I: Upper limit on $p_{a n n}$ from current WMAP observations and future upper limits achievable from the Planck satellite mission and from a cosmic variance limited experiment. 


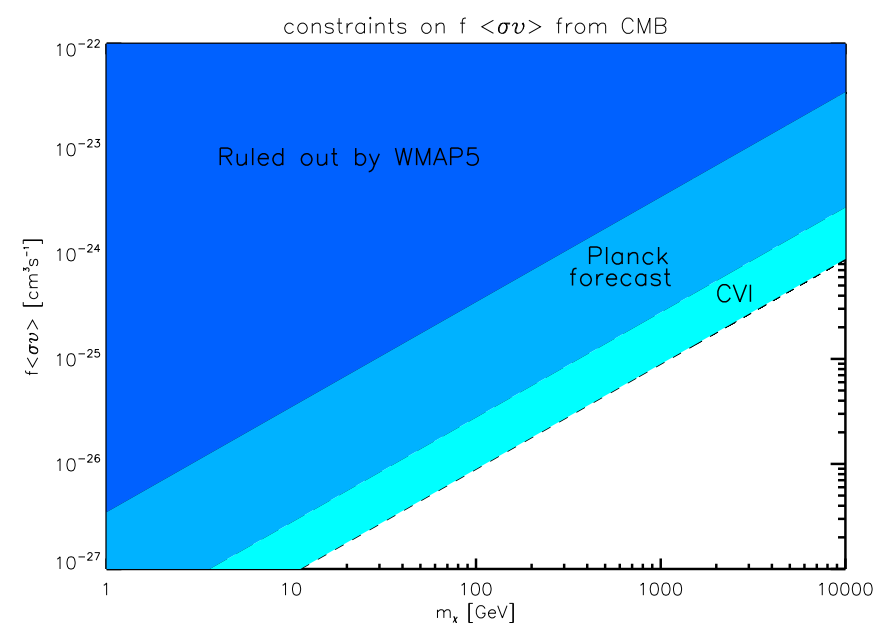

FIG. 4: Constraints on the self-annihilation cross-section at recombination $(\sigma v)_{z_{r}}$ times the gas-shower coupling parameter $f$. The dark blue area is already excluded by WMAP 5 data, whereas the more stringent limit (dashed area) refers to the constraints which will be possible to apply with Planck. The light blue area is the zone ultimately allowed to probe by a cosmic variance limited experiment with angular resolution comparable to Planck.

in the cosmomc package. We use a cosmic age top-hat prior as $10 \mathrm{Gyr} \leq t_{0} \leq 20 \mathrm{Gyr}$. We include the five-year WMAP data [1] (temperature and polarization) with the routine for computing the likelihood supplied by the WMAP team (we will refer to this analysis as WMAP5).

\section{RESULTS}

Using the WMAP-5 dataset and applying the analysis method described in the previous section, we found an upper limit $p_{a n n}<2.0 \times 10^{-6} \mathrm{~m}^{3} / \mathrm{s} / \mathrm{kg}$ at $95 \%$ c.l., with no indications for modified recombination in agreement with previous and similar analyses. The implications of this limit are discussed in the next section. While we detect only an upper limit it is interesting, from a cosmological point of view, to investigate the possible impact of this parameter on the estimation of other parameters as the baryon density $\omega_{b}$, the cold dark matter density $\omega_{c}$ and the scalar spectral index $n_{S}$. In Figure 3 we plot the 1-D likelihood distributions for these three parameters derived assuming the standard case (i.e. $p_{a n n}=0$ ) and letting this parameter to vary freely. As we can see, including $p_{\text {ann }}$ into the analysis changes the constraints of $\omega_{b}=0.0228 \pm 0.0006$ and $n_{s}=0.965 \pm 0.014$ (obtained in the standard case) to $\omega_{b}=0.0230 \pm 0.0006$ and $n_{s}=0.977 \pm 0.018$. The cosmological constraints on the cold dark matter density are on the contrary not affected by the inclusion of $p_{a n n}$.

With the advent of the Planck satellite mission, it is interesting to forecast to what extent the above limit will be improved by this mission. We have therefore forecasted future constraints on $p_{\text {ann }}$ assuming simulated Planck mock data with a fiducial model given by the best fit WMAP5 model (with standard recombination) and experimental noise described by (see [23]):

$$
N_{\ell}=\left(\frac{w^{-1 / 2}}{\mu \mathrm{K}-\mathrm{rad}}\right)^{2} \exp \left[\frac{\ell(\ell+1)\left(\theta_{\mathrm{FwHM}} / \mathrm{rad}\right)^{2}}{8 \ln 2}\right],
$$

with $w^{-1 / 2}=63 \mu K$ as the temperature noise level (we consider a factor $\sqrt{2}$ larger for polarization noise) and $\theta_{\text {FWHM }}=7^{\prime}$ for the beam size. We take $f_{\text {sky }}=0.65$ as sky coverage. We found that the Planck mission in the configuration described above will have the ability of placing a constraint of $p_{a n n}<1.5 \times 10^{-7} \mathrm{~m}^{3} / \mathrm{s} / \mathrm{kg}$ at $95 \%$ c.l.

It is also interesting to investigate the ultimate ability of cosmology to place constraints on $p_{a n n}$. We have therefore repeated the analysis with an ideal Cosmic Variance Limited experiment with resolution up to $\ell_{\max }=2500$. In this case we found $p_{a n n}<5.0 \times 10^{-8} \mathrm{~m}^{3} / \mathrm{s} / \mathrm{kg}$ at $95 \%$ c.l.

These constraints are summarized in fig. 4, where we show the allowed values of $f\langle\sigma v\rangle$ as a function of the WIMP mass $m_{\chi}$, for the different experiments described above. These results place useful constraints on the DM annihilation cross-section at very small relative velocity. This is particularly important for models with a large "Sommerfeld enhancement" (SE), a non-perturbative effect arising from the distortion of the wave functions of the two annihilating particles, due to the exchange of Coulomb-like forces mediated by (possibly new) force carriers 24]. The interest in these models arises from the fact that larger-than-thermal annihilation cross-section are required if one wants to explain the the rise in the electron and positron spectra observed by PAMELA and ATIC in terms of DM annihilation (see e.g. the discussion in Ref. 25]). We briefly recall here the basics of the SE. For two DM particles undergoing s-wave annihilation, the wave function in the non-relativistic limit obeys the Schrödinger equation

$$
\psi^{\prime \prime}(r)-m_{\chi} V(r) \psi(r)+m_{\chi}^{2} \beta^{2} \psi(r)=0
$$

In the limit where the mass of the carrier and the relative velocity of DM particles are small, it is easy to find an analytic approximation to the SE

$$
S(\beta)=\frac{\alpha \pi}{\beta}\left[1-\exp ^{-\alpha \pi / \beta}\right]
$$

which exhibits the $S \sim 1 / \beta$ behaviour that we mentioned in the introduction. Interestingly, a full calculation shows that the true solution saturates at $\beta \sim m_{\phi} / m_{\chi}$, and it actually develops resonances, that lead to very large SE for specific combinations of masses $m_{\phi}$ and $m_{\chi}$, and the coupling $\alpha$. In order to compare the constraints on $p_{\text {ann }}$ obtained from the analysis of CMB data with theoretical models, we have numerically integrated equation 13 , assuming a Yukawa potential $V(r)=-\exp \left[-m_{\phi} r\right] \alpha / r$ 


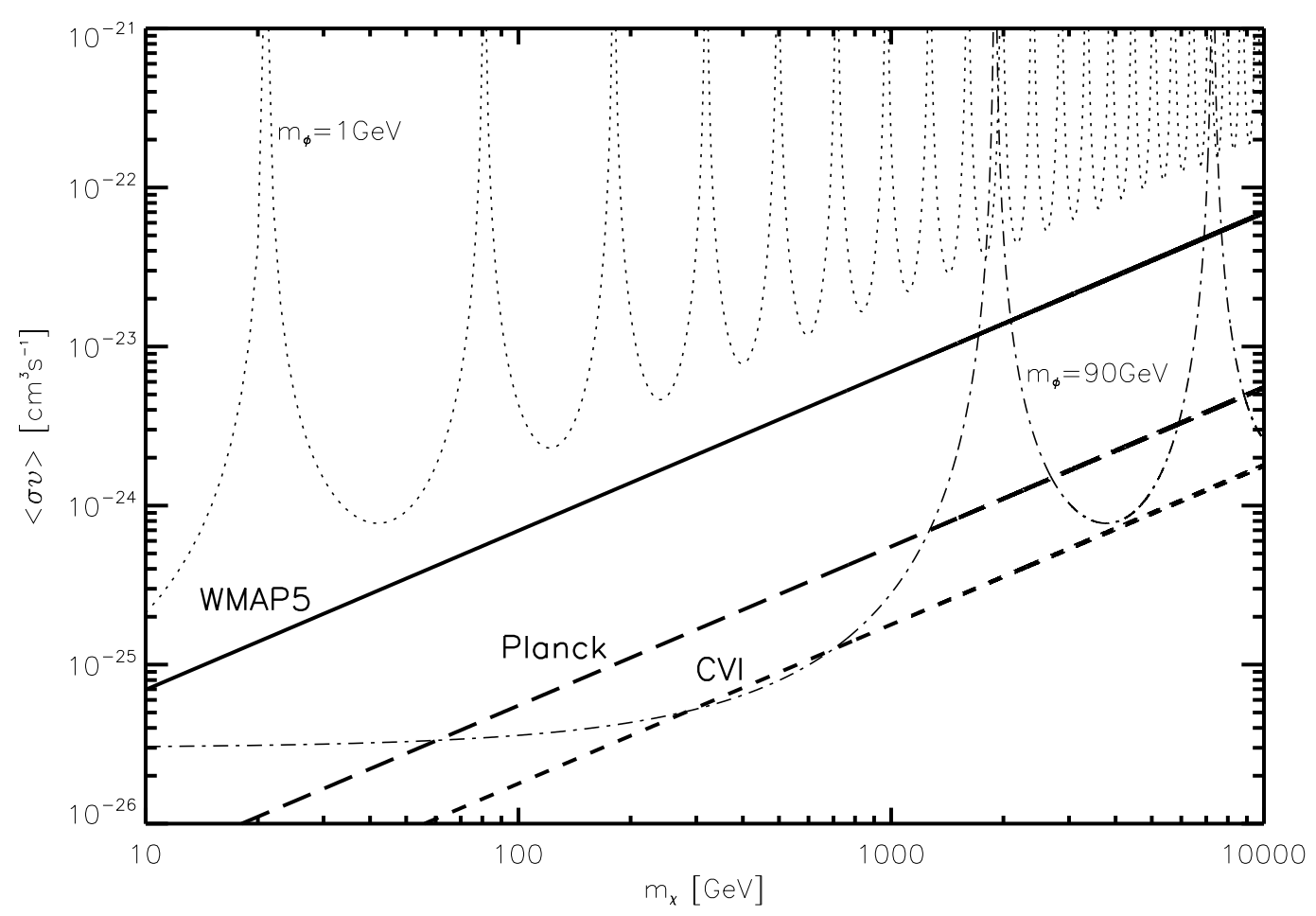

FIG. 5: Constraints on the self-annihilation cross-section at recombination $(\sigma v)_{z_{r}}$, assuming the gas-shower coupling parameter $f=0.5$, see text for details. Regions above the solid (/long dashed/short dashed) thick lines are ruled out by WMAP5 (/Planck forecast/Cosmic Variance limited); the thin dotted and dashed-dotted lines are the predictions of the "Sommerfeld" enhanced self-annihilation cross sections with force carrying bosons of $\mathrm{m}_{\phi}=1 \mathrm{GeV} / \mathrm{c}^{2}$ and $\mathrm{m}_{\phi}=90 \mathrm{GeV} / \mathrm{c}^{2}$ respectively, see text for details. Notice that these constraints apply to $\langle\sigma v\rangle$ at very low temperatures such that it is in saturated Sommerfeld regime, and therefore directly comparable with results from galatic substructures and dwarf galaxies constraints as from [12].

mediated by a boson of mass $m_{\phi}=1 \mathrm{GeV} / \mathrm{c}^{2}$, and $m_{\phi}=90 \mathrm{GeV} / \mathrm{c}^{2}$, taking $\alpha=1 / 4 \pi$ (see e.g. [26] for details); $\beta=10^{-8}$ as appropriate for the recombination epoch.

The results are visualized in fig.5, were we show the region excluded by our analysis in the $(\sigma v)$ vs. $m_{\chi}$ plane, corresponding to the $95 \%$ c.l. upper limit on the cross section that can be derived by combining eq. 11] with the constrains on $p_{a n n}$ in table 【

$\sigma v_{z_{r}, 26}^{\max }=71.2\left(\frac{p_{a n n}^{\max }}{2.0 \cdot 10^{-6} \mathrm{~m}^{3} \mathrm{~s}^{-1} \mathrm{~kg}^{-1}}\right)\left(\frac{m_{\chi}}{100 \mathrm{GeV}}\right)\left(\frac{0.5}{f}\right)$

where $\sigma v_{z_{r}, 26}^{\max }$ denotes the upper limit of the annihilation cross section at recombination in units of $10^{-26} \mathrm{~cm}^{3} \mathrm{~s}^{-1}$.

We have adopted in this formula, and in fig 5 a fiducial value $f=0.5$ for the coupling between the annihilation products and the gas, following the detailed calculation of DM-induced shower propagation and energy release performed by [27]; this number is a good approximation averaged on all channels, although its actual value will ultimately depend on the composition of the shower and on its energy spectrum, namely on the nature of the annihilating DM particle itself. It is however straightforward to obtain the constraints for different values of $f$.
We find that the most extreme enhancements are already ruled out by existing CMB data, while enhancements of order $10^{3}-10^{4}$ with respect to thermal value $\langle\sigma v\rangle=3 \times 10^{-26} \mathrm{~cm}^{3} / \mathrm{s}$, required to explain the PAMELA and ATIC data, will be probed over a larger WIMP mass range by Planck. We also note that for small enough $m_{\chi}$, a CMB experiment allows us to probe the region of thermal cross-sections, and that Planck sensitivity will reach it, making it possible perhaps to find hints of particle $\mathrm{DM}$ in CMB data.

We note that the constraints obtained here are several orders of magnitude more stringent than those obtained from the analysis of high-redshift proto-halos [13] (see also the recent [28]). We stress that our results apply also for standard models with s-wave annihilations, where the annihilation cross section does not depend on $v$. In this case, our results can be directly compared with the constraints from astrophysical observations in the local universe [29]. 


\section{CONCLUSIONS}

We have studied the effects of WIMP DM selfannihilation on recombination, looking for signatures in the CMB anisotropy and polarization. Our analysis has been performed under the assumption that the shower produced by the WIMP annihilation interacts "locally" with plasma, and a fraction $f$ of the energy is absorbed on-the-spot by the baryons, contributing to its ionization. Our methodology is consistent with other analysis on the effect of decaying or self-annihilating, low-mass dark matter performed in the past. We examine a range of higher WIMP masses $\left(1 \mathrm{GeV} / \mathrm{c}^{2}-10 \mathrm{TeV} / \mathrm{c}^{2}\right)$, and find that current WMAP data already allow us to put interesting constraints on self-annihilation cross sections higher than the "standard" thermal value, in the range of those required to explain the PAMELA and ATIC data in terms of dark matter. Physically motivated by the very low relative velocity of DM particles at the time of recombination, our constraints on "Sommerfeld" enhanced cross sections are competitive with the existing ones from local Universe (galactic substructures), and an independent test achieved with standard physics of the early Universe. By using simulated mock data, we have found that the expected enhanced sensitivity of the Planck mission will improve our capability to constrain Sommerfeld enhancement in dark matter particle models, thus hinting toward the exciting possibility to be finding traces of particle dark matter in future CMB data. Interestingly, Planck will very likely be able to probe the region of the thermal annihilation cross-section for low WIMP masses $\left(\lesssim 50 \mathrm{GeV} / c^{2}\right)$, the actual value depending on the gas-shower coupling $f$. Ultimately, a cosmic variance limited experiment permits the possibility to probe cross-sections values at the order of the thermal one for $\mathcal{O}\left(100 \mathrm{GeV} / \mathrm{c}^{2}\right)$ mass WIMPs.

\section{Acknowledgments}

We are glad to acknowledge fruitful conversations with Marco Cirelli, Douglas Finkbeiner and Pasquale D. Serpico. S. G. and A. M. would like to thank M. Kamionkowski for useful comments. This research has been partially supported by ASI contract I/016/07/0 "COFIS."
[1] E. Komatsu et al., arXiv:0803.0547 [astro-ph].

[2] C. L. Reichardt et al., "High resolution CMB power spectrum from the complete ACBAR data set," arXiv:0801.1491 [astro-ph].

[3] T. E. Montroy et al. [BOOMERanG Collaboration], Astrophys. J. 647, 813 (2006) arXiv:astro-ph/0507514.

[4] P.J.E. Peebles, S. Seager, W. Hu, Astrophys. J. 539, L (1) (2000), astro-ph/0004389;

[5] S. Galli, R. Bean, A. Melchiorri and J. Silk, Phys. Rev. D 78 (2008) 063532 arXiv:0807.1420 [astro-ph]]; R. Bean, A. Melchiorri and J. Silk, Phys. Rev. D 75 (2007) 063505 arXiv:astro-ph/0701224; R. Bean, A. Melchiorri and J. Silk, Phys. Rev. D 68 (2003) 083501 arXiv:astro-ph/0306357.

[6] J. Kim and P. Naselsky, arXiv:0802.4005 [astro-ph].

[7] A. Lewis, J. Weller, and R. Battye, Mon. Not. Roy. Astron. Soc. 373, 561 (2006) arXiv:astro-ph/0606552.

[8] A. G. Doroshkevich and P. D. Naselsky, Phys. Rev. D 65, 123517 (2002) arXiv:astro-ph/0201212 ; P. D. Naselsky and L. Y. Chiang, Phys. Rev. D 69, 123518 (2004) arXiv:astro-ph/0312168; E. Pierpaoli, Phys. Rev. Lett. 92, 031301 (2004) arXiv:astro-ph/0310375]; X. L. Chen and M. Kamionkowski, Phys. Rev. D 70, 043502 (2004) arXiv:astro-ph/0310473; N. Padmanabhan and D. P. Finkbeiner, Phys. Rev. D 72, 023508 (2005) arXiv:astro-ph/0503486]; M. Mapelli, A. Ferrara and E. Pierpaoli, Mon. Not. Roy. Astron. Soc. 369, 1719 (2006) arXiv:astro-ph/0603237.

[9] L. Zhang, X. L. Chen, Y. A. Lei and Z. G. Si, Phys. Rev. D 74, 103519 (2006) arXiv:astro-ph/0603425; L. Zhang, X. Chen, M. Kamionkowski, Z. g. Si and Z. Zheng, Phys. Rev. D 76, 061301 (2007) arXiv:0704.2444 [astro-ph]].

[10] O. Adriani et al. [PAMELA Collaboration], Nature 458, 607 (2009) arXiv:0810.4995 [astro-ph]].
[11] J. Chang et al., Nature 456, 362 (2008).

[12] M. Lattanzi and J. I. Silk, arXiv:0812.0360 [astroph]; L. Pieri, M. Lattanzi and J. Silk, arXiv:0902.4330 [astro-ph.HE]; R. Essig, N. Sehgal and L. E. Strigari, arXiv:0902.4750 [hep-ph].

[13] M. Kamionkowski and S. Profumo, Phys. Rev. Lett. 101, 261301 (2008) arXiv:0810.3233 [astro-ph]].

[14] M. Cirelli, M. Kadastik, M. Raidal and A. Strumia, Nucl. Phys. B 813 (2009) 1 arXiv:0809.2409 [hep-ph]]; L. Bergstrom, G. Bertone, T. Bringmann, J. Edsjo and M. Taoso, arXiv:0812.3895 [astro-ph]; G. Bertone, M. Cirelli, A. Strumia and M. Taoso, JCAP 0903 (2009) 009 arXiv:0811.3744 [astro-ph]]; F. Donato, D. Maurin, P. Brun, T. Delahaye and P. Salati, Phys. Rev. Lett. 102 (2009) 071301 arXiv:0810.5292 [astro-ph]]; M. Fornasa, L. Pieri, G. Bertone and E. Branchini, arXiv:0901.2921 [astro-ph].

[15] P.J.E. Peebles, Astrophys. J. 153, 1 (()1968). Ya. B. Zel'dovich, V.G. Kurt, R.A. Sunyaev, Zh. Eksp. Teoret. Fiz 55 278(1968), English translation, Sov. Phys. JETP. 28146 (1969).

[16] X. L. Chen and M. Kamionkowski, Phys. Rev. D 70, 043502 (2004) arXiv:astro-ph/0310473.

[17] Shull, J. M.; van Steenberg, M. E. Astrophys. J. 298, 2 (6)8 (1985)

[18] S. Seager, D. D. Sasselov and D. Scott, Astrophys. J. Suppl. 128, 407 (2000) arXiv:astro-ph/9912182.

[19] A. Lewis, A. Challinor and A. Lasenby, Astrophys. J. 538, 473 (2000) arXiv:astro-ph/9911177.

[20] A. Natarajan and D. J. Schwarz, arXiv:0903.4485 [astroph.CO];

[21] A. V. Belikov and D. Hooper, arXiv:0904.1210 [hep-ph].

[22] A. Lewis and S. Bridle, Phys. Rev. D 66, 103511 (2002) (Available from http://cosmologist.info.) 
[23] [Planck Collaboration], arXiv:astro-ph/0604069.

[24] A. Sommerfeld, Ann. Phys. 11257 (1931). J. Hisano, S. Matsumoto and M. M. Nojiri, Phys. Rev. Lett. 92 (2004) 031303 hep-ph/0307216. J. Hisano, S. Matsumoto, M. M. Nojiri and O. Saito, Phys. Rev. D 71 (2005) 015007 hep-ph/0407168.

[25] M. Cirelli, M. Kadastik, M. Raidal and A. Strumia, Nucl. Phys. B 813 (2009) 1 arXiv:0809.2409 [hep-ph]].

[26] N. Arkani-Hamed, D. P. Finkbeiner, T. R. Slatyer and N. Weiner, Phys. Rev. D 79, 015014 (2009) arXiv:0810.0713 [hep-ph]].
[27] D. Finkbeiner, [Private Communication]

[28] F. Y. Cyr-Racine, S. Profumo and K. Sigurdson, arXiv:0904.3933 [astro-ph.CO].

[29] N. F. Bell and T. D. Jacques, arXiv:0811.0821 [astro-ph]; N. F. Bell, J. B. Dent, T. D. Jacques and T. J. Weiler, Phys. Rev. D 78 (2008) 083540 arXiv:0805.3423 [hepph]]; G. D. Mack, T. D. Jacques, J. F. Beacom, N. F. Bell and H. Yuksel, Phys. Rev. D 78 (2008) 063542 arXiv:0803.0157 [astro-ph]]. 